\title{
Real-time Investigation of Nanoparticle Self-assembly Mechanisms and Its Controlling Factors
}

\author{
Jaewon Lee ${ }^{1}$, Elias Nakouzi ${ }^{1}$, Jaehun Chun ${ }^{1}$ and Dongsheng $\mathrm{Li}^{1 *}$ \\ 1. Physical and Computational Sciences Directorate, Pacific Northwest National Laboratory, Richland, \\ WA, USA. \\ * Corresponding author: Dongsheng.Li2@pnnl.gov
}

Superlattice structures formed by nanoparticle (NP) self-assembly have attracted increasing attention due to their potential as a novel class of nanomaterials with enhanced physicochemical properties tailored by the assembly structure.[1] For instance, rationally designing superlattice patterns with defined separation distances can play an important role in controlling properties such as surface plasmon resonance. However, many key questions remain regarding the correlation between the dynamics of individual NPs and the emerging superlattice patterns. Such self-assemblies directly result from a subtle balance between various forces between NPs over different length scales. Brownian force $\left(F_{B r}\right)$ resulting from thermal energy triggers motions of NPs. The van der Waals force $\left(F_{v d W}\right)$ between NPs is generally a major attractive force at close separations, whereas the hydrodynamic force $\left(\mathrm{F}_{\mathrm{D}}\right)$, electrostatic force $\left(F_{\text {elec }}\right)$, hydration force $\left(F_{h y}\right)$, and steric hindrance force $\left(F_{S h}\right)$ are repulsive forces.

To gain insights into the self-assembly process, we conducted in situ liquid cell TEM (LCTEM) experiments (Fig. 1 and 2) and monitored the silver or gold NP assembly process in real time to reveal the kinetics and the driving sources of assembly process under various experimental conditions, such as solvents and ligands.

To understand kinetic details of the process, the self-assembly of gold NPs are further analyzed by calculating the contributions of various forces involved [3-4]. Our results show that these forces, including $F_{B r}, F_{v d W}, F_{e l}$, and $F_{D}$, and their interplay play a key role in NP self-assembly process. The superlattice structure can be controlled via solvents or ligands.

These results provide insights into the complex competition between forces at different scales. The obtained knowledge of interactions between surface coating materials can be extended to a wide range of ligands with different chain lengths, functionalized groups, or biodegradable polymers that can be applied to the fields of material synthesis, bio-mineralization, bio-sensors, or electronic devices.

\section{References:}

[1] M Boles, M Engel and DV Talapin. Chemical Reviews 116 (2016), p. 11220.

[2] JM Yuk et al., Science 336 (2012), p. 61.

[3] W.B. Russel et al., in "Colloidal dispersions", Ed. GK Batchelor (Cambridge University Press, New York) p. 1.

[4] JN Israelachvili, in "Intermolecular and Surface Forces", Academic Press, (Elsevier, Santa Barbara) p. 3. 

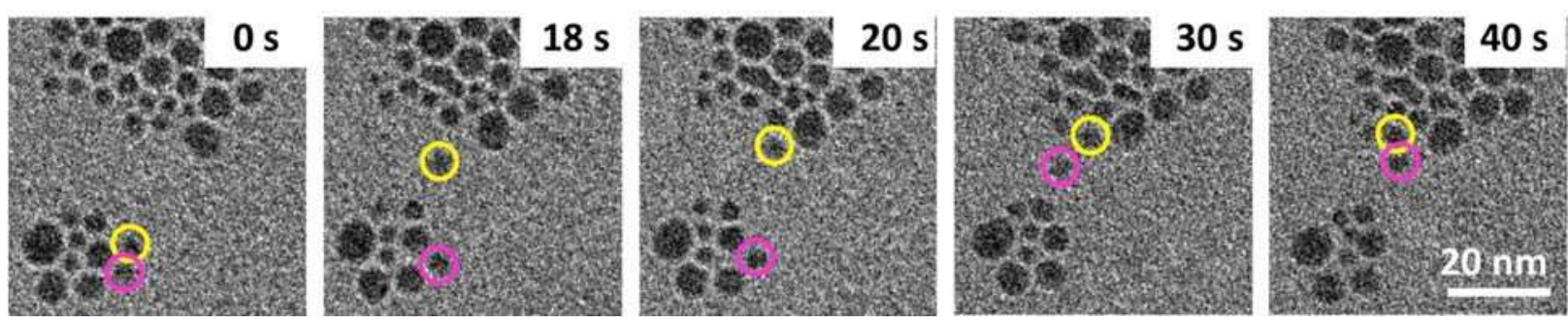

Figure 1. Snapshots of in situ LCTEM images of silver NPs. Yellow and red highlighted particles moved from a small cluster to a large cluster.

(a) Repulsive Forces:

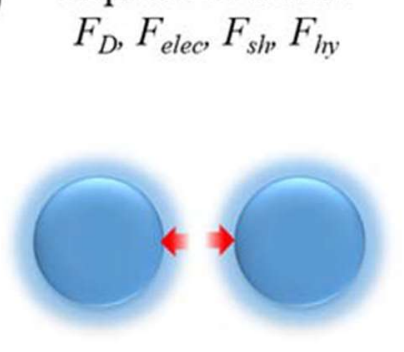

$$
F_{D}, F_{\text {eleo }}, F_{s h}, F_{l y}
$$

(b)

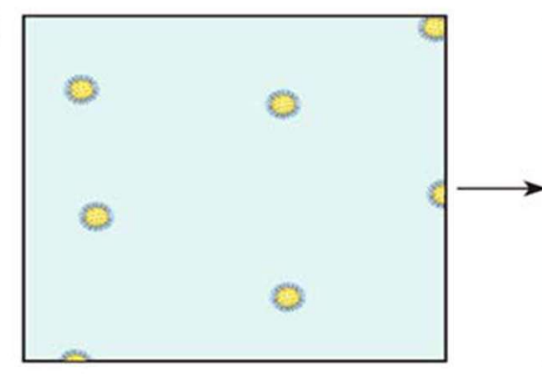

Attractive Fore:

$$
F_{v d W}
$$
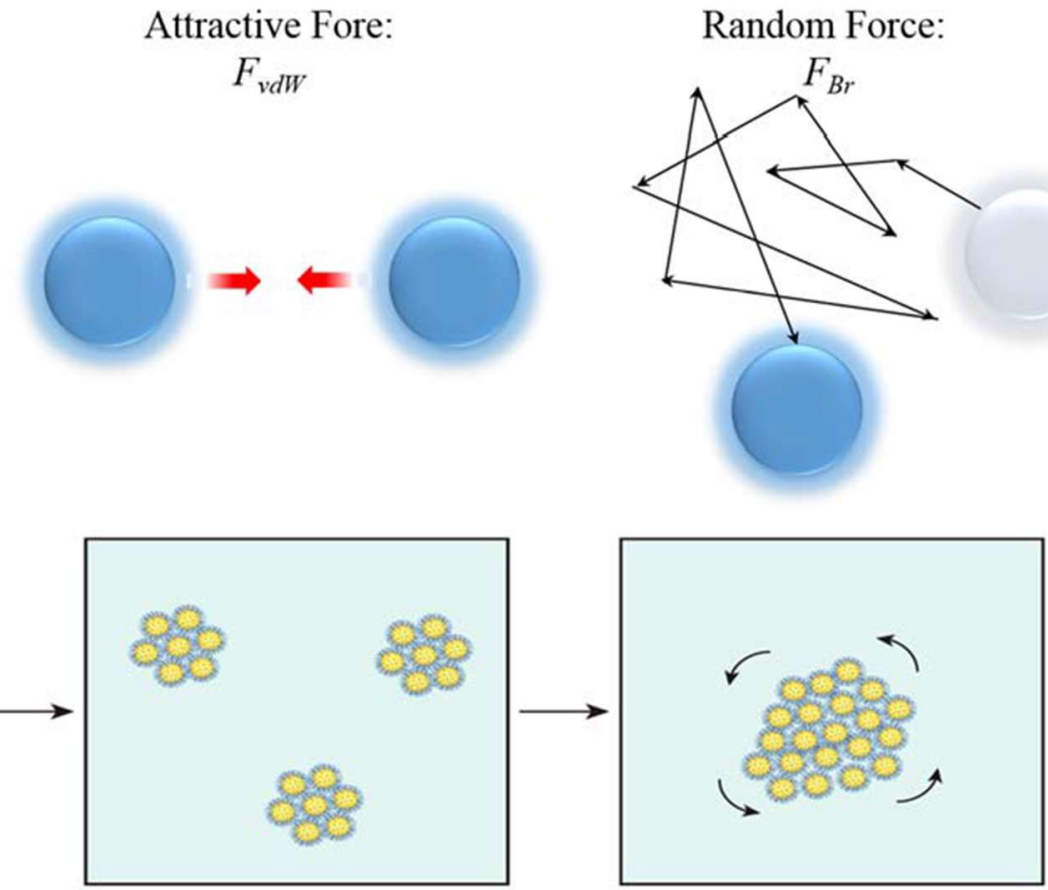

Figure 2. Schematic drawing of (a) particle-particle force interactions and (b) self-assembly process of NPs as a function of time. $F_{D}$ is the hydrodynamic force, $F_{\text {elec }}$ is the electrostatic force, $F_{\text {sh }}$ is the steric hindrance force, $F_{h y}$ is the hydration force, $F_{v d W}$ is the van der Waals force and $F_{B r}$ is the Brownian force. 